\title{
Technical note: Estimating body weight of dairy calves with a partial-weight scale attached to an automated milk feeder
}

\author{
Melissa C. Cantor, ${ }^{1}$ (1) Charlotte H. Pertuisel, ${ }^{2}$ and Joao H. C. Costa ${ }^{1 *}$ (1) \\ ${ }^{1}$ Department of Animal and Food Sciences, University of Kentucky, Lexington 40546 \\ ${ }^{2}$ AgroParisTech, Paris, France, 70005
}

\begin{abstract}
Monitoring early growth in neonatal dairy calves provides insight into the effectiveness of a producer's nutrition program and helps monitor for sickness. The objective of this study was to validate whether a partial-weight scale attached to an automated milk feeder (Combi; DeLaval, Tumba, Sweden) could precisely and accurately estimate calf weights compared with twice-weekly weights of a calibrated electronic scale (gold standard; Brecknell PS1000; Avery WeighTronix LLC, Fairmont, MN). Holstein heifers $(\mathrm{n}=20)$ were enrolled in this study from birth until $84 \mathrm{~d}$ of age. All heifers were eligible to receive $10 \mathrm{~L}$ of milk replacer/d for $56 \mathrm{~d}$ and were subsequently weaned using a step-down strategy across $14 \mathrm{~d}$. The automated milk feeder had a radio frequency identification panel placed directly above the scale such that calves had to stand with both front hooves on the scale platform to access milk from the nipple. An algorithm was created to summarize and clean the scale measurements of any non-biologically relevant data points. The relationship between the daily weight average from the partial scale and the electronic scale was analyzed using Pearson correlations, linear regressions, and Bland-Altman plots. Data from the partial weight scale were considered precise if the correlation coefficient and coefficient of determination were very high $(>0.90)$ and the mean bias from the Bland-Altman plots included zero with the $95 \%$ interval of agreement. The partial-weight scale was considered accurate if the slope from the linear regression did not differ significantly from 1 . We found a very high Pearson correlation coefficient (0.99) and coefficient of determination (0.99). Bland-Altman plots were deemed acceptable and nonbiased; the BlandAltman difference (feeder weight - scale weight) was $0.45 \pm 2.33 \mathrm{~kg}$ (mean \pm standard deviation). The slope of the linear regression was not different from 1 (slope
\end{abstract}

Received May 6, 2019.

Accepted October 14, 2019

*Corresponding author: costa@uky.edu
$=1.01 ; 95 \%$ confidence interval $=1.00-1.03)$, suggesting that the partial-weight scale was accurate. In summary, the partial-weight scale was validated, with a high precision and accurate estimate of weight in calves compared with the gold standard electronic scale. The partial-weight scale may be a useful tool for producers to estimate calf weight if a data cleaning algorithm is used.

Key words: average daily gain, calf growth, precision dairy technology, preweaning

\section{Technical Note}

Monitoring early growth in heifers helps monitor for sickness (Enevoldsen and Kristensen, 1997), assess the success of the nutritional program (Bach and Ahedo, 2008), and achieve long-term sufficient growth for calving at 24 mo of age (Radostits et al., 1994). Visual estimation of live weight in heifers is highly variable; in fact, accuracy of live estimation of heifer BW decreases as the animal increases in size, and weight less than 150 $\mathrm{kg}$ in young animals tends to be overestimated (Wood et al., 2015). Visual weight estimates likely vary due to variation in experience and skill of the individual, so other means of monitoring weights are needed. The most accurate method for measuring weight is using a calibrated electronic scale; this is considered the gold standard (Dingwell et al., 2006). However, the use of electronic scales is labor intensive (Heinrichs et al., 1992) and a costly initial investment (Heinrichs et al., 1992). One indirect method for estimating weight in heifers is with a heart girth tape, which has an equation that has been validated over time (Heinrichs et al., 1992, 2017). However, heart girth tapes are also labor intensive (Heinrichs et al., 2017) and likely require training to avoid human error. Another labor-intensive alternative is the hoof circumference tape; however, it is validated only for estimating weight of dairy calves between 31 and $45 \mathrm{~kg}$ (Long et al., 2012). Research has also shown that hipometers, which estimate heifer growth based on the width between the hips and greater trochanters of the femurs, have a poor measurement agreement 
between animals younger than 3 mo and older than 15 mo of age (Dingwell et al., 2006). Therefore, there is a need for better indirect measurements for estimating weight in dairy calves.

One potential alternative technology is an automated scale that reduces the labor required to manually weigh calves. One such automated scale is a partial scale attached to an automated milk feeder; weights are automatically recorded and processed by the feeder's software, and a daily average weight is reported for each individual. To our knowledge, these partial-weight scales attached to automated feeders have not been validated in the peer-reviewed literature. To be recommended for a commercial setting, these partial-weight scales should be validated by comparing them with an electronic scale (the gold standard). A high precision and accuracy of estimated calf weights is necessary because these weights are often used to identify whether a calf is sick and losing weight, to determine proper dosage for medications for treatment decisions, and to evaluate the effectiveness of nutritional programs over time. Therefore, the objective of this study was to validate the precision and accuracy of a partial-weight scale attached to an automated milk feeder in estimating weight in young calves compared with a calibrated electronic scale.

We enrolled female Holstein dairy calves $(\mathrm{n}=20)$, with 19 weights per calf and a total number of 405 recorded weights. Due to the cleaning algorithm, 376 weights were considered in the final analyses. All heifers born at the University of Kentucky Coldstream Dairy Research farm between June 15 and October 6, 2018, were enrolled in this study, and no heifers were excluded. All heifers were part of the Institutional Animal Care and Use Committee approval code 2018:2864. As described in Moinester and Gottfried (2014), for Pearson correlation values of $\mathrm{r}=0.80$ and half-widths of $\mathrm{w}=$ 0.05 , a sample size of 205 was needed to have power to detect a difference. We enrolled several heifers required to have power to test whether the partial-weight scale estimated weights consistently through time and to ensure that various calf weights were represented. Birth weights were taken on all heifers using an electronic scale (gold standard; Brecknell PS1000, Avery WeighTronix LLC, Fairmont, MN). Heifers at birth weighed (mean $\pm \mathrm{SD}) 35.04 \pm 6.04 \mathrm{~kg}$. Calves were enrolled at $5 \pm 2 \mathrm{~d}$ of age and placed in a pen $(4.57 \times 10.67$ m) with a partial scale attached to an automated milk feeder (Combi; DeLaval, Tumba, Sweden) and starter feeder (DeLaval) containing $6 \pm 3$ other heifers and bedded with sawdust shavings. To reduce automated milk feeder competition, calves were moved to an identical adjacent pen with the same stocking density before weaning. Therefore, 2 partial-weight scales were used in this study, but both were associated with the same automated milk calf feeder. Calves remained in the study until $86 \pm 4 \mathrm{~d}$ of age and weighed $100.04 \pm$ $17.04 \mathrm{~kg}$ at the end of the study. The automated milk feeder had a radio frequency identification panel placed directly above the scale such that calves had to stand with both front hooves on the scale platform to access milk from the nipple. Calves were fed the first meal with human assistance and received human assistance every $12 \mathrm{~h}$ until independent milk consumption was observed. Calves were allotted up to $10 \mathrm{~L}$ of milk replacer/d (Cow's Match; Land O' Lakes Animal Milk Products Co., Shoreview, MN) for 50 feeder days, reduced to $50 \%$ allotment for 14 feeder days, and then reduced to $20 \%$ allotment for an additional 7 feeder days until weaning at 70 feeder days. Milk replacer on a percentage DM basis was a minimum $28 \% \mathrm{CP}, 20 \%$ fat, a maximum $15 \%$ crude fiber, a minimum $1.0 \%$ calcium and $0.70 \%$ phosphorus, and contained $4.87 \mathrm{Mcal}$ of $\mathrm{ME} / \mathrm{kg}$. Calves were followed for $14 \mathrm{~d}$ after weaning. The automated starter feeder contained a 22\% CP starter (Calf Starter; Baghdad Feeds, Shelby, KY). Calves were also offered chopped alfalfa in a trough $(1.83 \times 0.33 \times 0.16 \mathrm{~m})$, which was sampled once weekly and averaged (\% DM basis) $90.5 \%$ DM, $13.4 \%$ protein, $51.1 \%$ ADF, $61.5 \%$ NDF, $12.9 \%$ ash, and $0.7 \%$ crude fat. All calves had ad libitum access to water, starter, and chopped alfalfa hay during the study period.

The partial-weight scale recorded calf weight every time the calf visited the feeder and automatically calculated the daily average weight $(\mathrm{kg})$ for each calf, which was recorded in the feeder computer. For comparison, calves were weighed every Monday and Thursday weekly until 2 wk postweaning on the same electronic scale used for obtaining birth weights.

All statistical analyses were performed in R (version 3.5.2 and RStudio version 1.2.5001, RStudio Inc., Boston, MA). For cleaning the partial-weight data, an algorithm was created to summarize and clean the scale measurements of any non-biologically relevant data points. The cleaning algorithm removed measurements of zero weight $(19 / 405)$, measurements lower than the first week weight $(4 / 405)$, or measurements that deviated more than $3 \mathrm{SD}$ or $15 \%$ from the weight average of the 3 previous days $(6 / 405)$. The standard deviation threshold was created by building an algorithm for every $0.5 \mathrm{SD}$ starting at $1 \mathrm{SD}$ and for every $5 \%$ change from $10 \%$. The $3 \mathrm{SD}$ was the most efficient cleaning algorithm, where the coefficient of determination minimized the loss of data points. In total, $7 \%$ (29/405) of data points were removed, with 376 points included for analysis.

Precision was defined by Pearson correlation coefficients and a linear regression coefficient of determina- 
Table 1. The linear regression relationship of each dairy heifer $(n=20)$ weight estimated using a partialweight scale attached to an automated milk feeder and weights measured manually using an electronic scale, including number of measurements $(\mathrm{N})$, coefficient of determination, intercept, lower and upper $95 \%$ confidence intervals, and slope

\begin{tabular}{|c|c|c|c|c|c|c|c|c|}
\hline \multirow[b]{2}{*}{ Calf ID } & \multirow[b]{2}{*}{$\mathrm{N}$} & \multirow[b]{2}{*}{$\mathrm{R}^{2}$} & \multirow[b]{2}{*}{ Intercept } & \multicolumn{2}{|c|}{$95 \%$ CI } & \multirow[b]{2}{*}{ Slope } & \multicolumn{2}{|c|}{$95 \%$ CI } \\
\hline & & & & Lower & Upper & & Lower & Upper \\
\hline 1932 & 19 & 0.98 & -8.09 & -13.19 & -2.98 & 1.12 & 1.04 & 1.19 \\
\hline 1933 & 19 & 0.93 & 5.9 & -2.82 & 14.62 & 0.88 & 0.76 & 1 \\
\hline 1935 & 17 & 0.93 & 11.23 & 1.55 & 20.9 & 0.82 & 0.69 & 0.96 \\
\hline 1936 & 19 & 0.98 & -5.3 & -10.31 & -0.28 & 1.06 & 0.99 & 1.14 \\
\hline 1937 & 19 & 0.98 & -5.5 & -10.63 & -0.37 & 1.06 & 0.99 & 1.13 \\
\hline 1942 & 19 & 0.99 & -6.74 & -10.05 & -3.43 & 1.09 & 1.04 & 1.14 \\
\hline 1943 & 19 & 0.98 & -6.41 & -11.5 & -1.31 & 1.07 & 1 & 1.14 \\
\hline 1944 & 19 & 0.97 & 1.88 & -2.55 & 6.31 & 0.95 & 0.87 & 1.03 \\
\hline 1945 & 19 & 0.99 & -8 & -11.84 & -4.16 & 1.11 & 1.05 & 1.17 \\
\hline 1947 & 19 & 0.99 & 1.77 & -1.31 & 4.84 & 1 & 0.95 & 1.04 \\
\hline 1948 & 18 & 0.98 & 2.93 & -2.08 & 7.95 & 0.94 & 0.87 & 1.01 \\
\hline 1949 & 19 & 0.99 & -0.85 & -4.79 & 3.08 & 1 & 0.95 & 1.06 \\
\hline 1950 & 19 & 0.99 & -1.21 & -3.68 & 1.26 & 1.01 & 0.98 & 1.05 \\
\hline 1951 & 19 & 0.97 & 1.71 & -3.7 & 7.11 & 0.99 & 0.91 & 1.08 \\
\hline 1952 & 19 & 0.99 & -2.38 & -5.75 & 0.99 & 1.03 & 0.98 & 1.08 \\
\hline 1953 & 19 & 0.98 & -4.05 & -8.27 & 0.16 & 1.09 & 1.02 & 1.17 \\
\hline 1954 & 19 & 0.99 & -1.34 & -5.49 & 2.81 & 1.03 & 0.97 & 1.09 \\
\hline 1955 & 19 & 0.99 & -3.17 & -6.52 & 0.19 & 1.02 & 0.98 & 1.07 \\
\hline 1956 & 18 & 0.99 & 0.39 & -4.11 & 4.9 & 0.98 & 0.92 & 1.05 \\
\hline 1957 & 19 & 1 & -0.63 & -2.14 & 0.89 & 1.01 & 0.99 & 1.03 \\
\hline
\end{tabular}

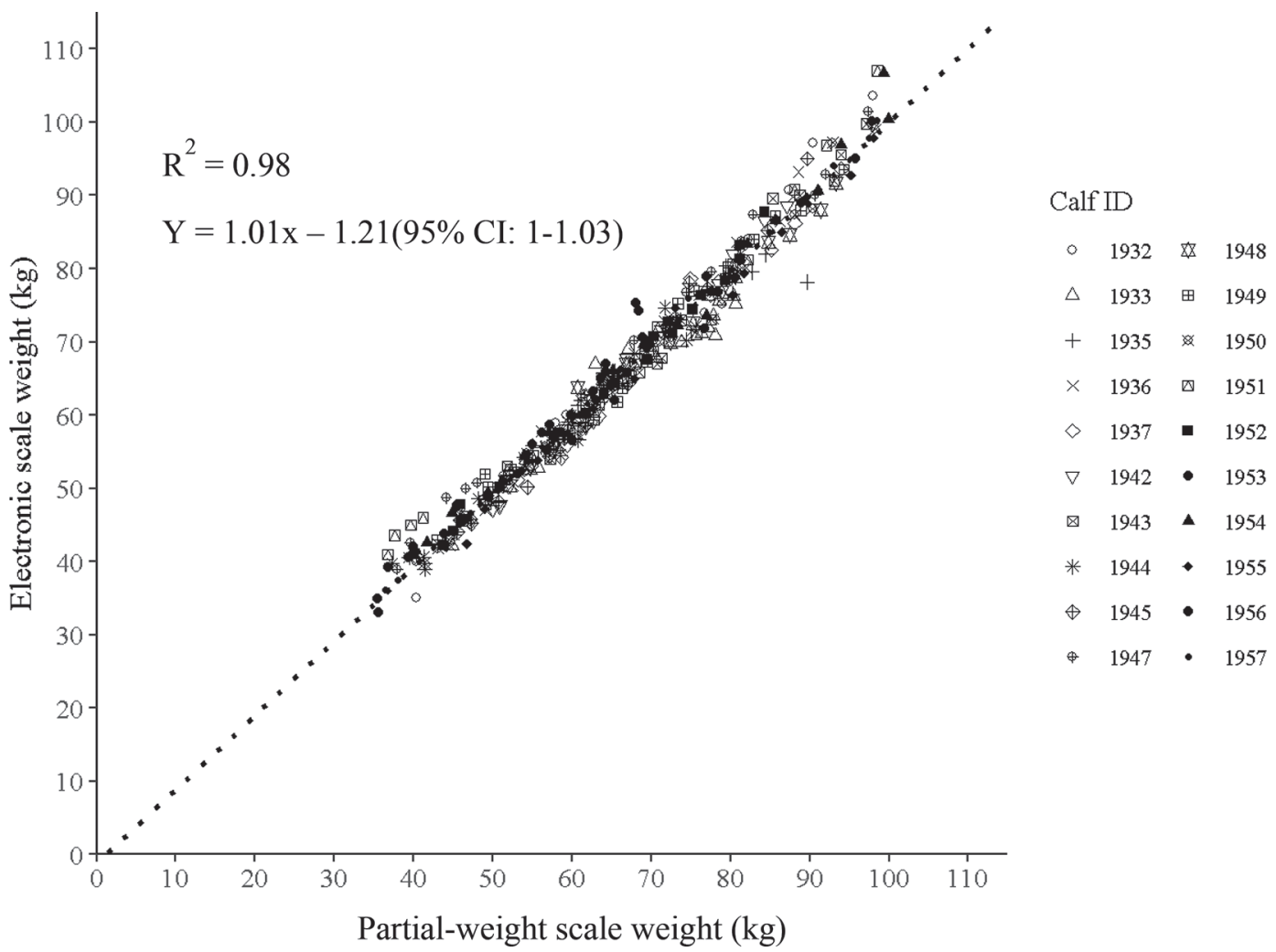

Figure 1. The linear regression relationship of dairy heifer $(n=20)$ weights $(376$ observations) estimated using a partial-weight scale attached to an automated milk feeder (x-axis) and weights measured manually using an electronic scale (y-axis). All heifers had 19 weights except for 1935 (17 weights), 1948 (18 weights), and 1956 (18 weights). 
tion and categorized following Hinkle (1988; 0.00-0.30 $=$ namely negligible; $0.30-0.50=$ low; $0.50-0.70=$ moderate; $0.70-0.90=$ high; $0.90-1.00=$ very high). Data from the partial-weight scale were considered precise if the correlation coefficient and coefficient of determination were very high $(>0.90)$. Accuracy was assessed by comparing the slopes of the linear regressions of the partial-weight scale and the electronic scale calf weights and using Bland-Altman plots. The x-axis showed the mean of the partial-weight scale and electronic scale weights [(partial-weight scale + electronic scale)/2)] for each of the 20 calves plotted (Table 1 ). The $\mathrm{y}$-axis showed the difference of the weight compared with the electronic scale weight (feeder weight - scale weight) for each of the 20 calves plotted. The feeder scale was considered accurate if the slopes of the linear regressions did not differ significantly from 1 and if the $95 \%$ interval of agreement included zero for mean bias from the Bland-Altman plots. A regression of each calf's weight against the partial-weight scale was created to determine whether individual calf had an effect and to ensure proper reporting of precision and accuracy. In addition, a paired $t$-test was run to assess for weight differences between the partial-weight scale and the electronic scale.

The Pearson correlation coefficient between partial scale calf weights and the electronic scale was very high $(\mathrm{r}=0.99 ; P<0.001)$, and the linear equation ( $\mathrm{y}=$ $1.01 \mathrm{x}-1.21)$ had a very high coefficient of determination $\left(\mathrm{R}^{2}=0.99 ; P<0.001 ;\right.$ Figure 1$)$. The slope of the regression was 1.01 (95\% CI: 1-1.03). The $95 \%$ confidence interval of the Bland-Altman plot (Figure 2) encompassed most of the observations, and most of them were distributed around 0. A linear regression of each calf's weights between the 2 scales across time is presented in Figure 3, and coefficients of determination, equations, and slopes are reported in Table 1 . The mean differences in weights (using a paired $t$-test) between the partial-weight scale and the electronic scale were $0.45 \pm 0.21 \mathrm{~kg}(P<0.001)$.

We observed high precision with the partial scale, with a very high Pearson correlation coefficient and a very high coefficient of determination. Precision indicates how closely measures are to one another

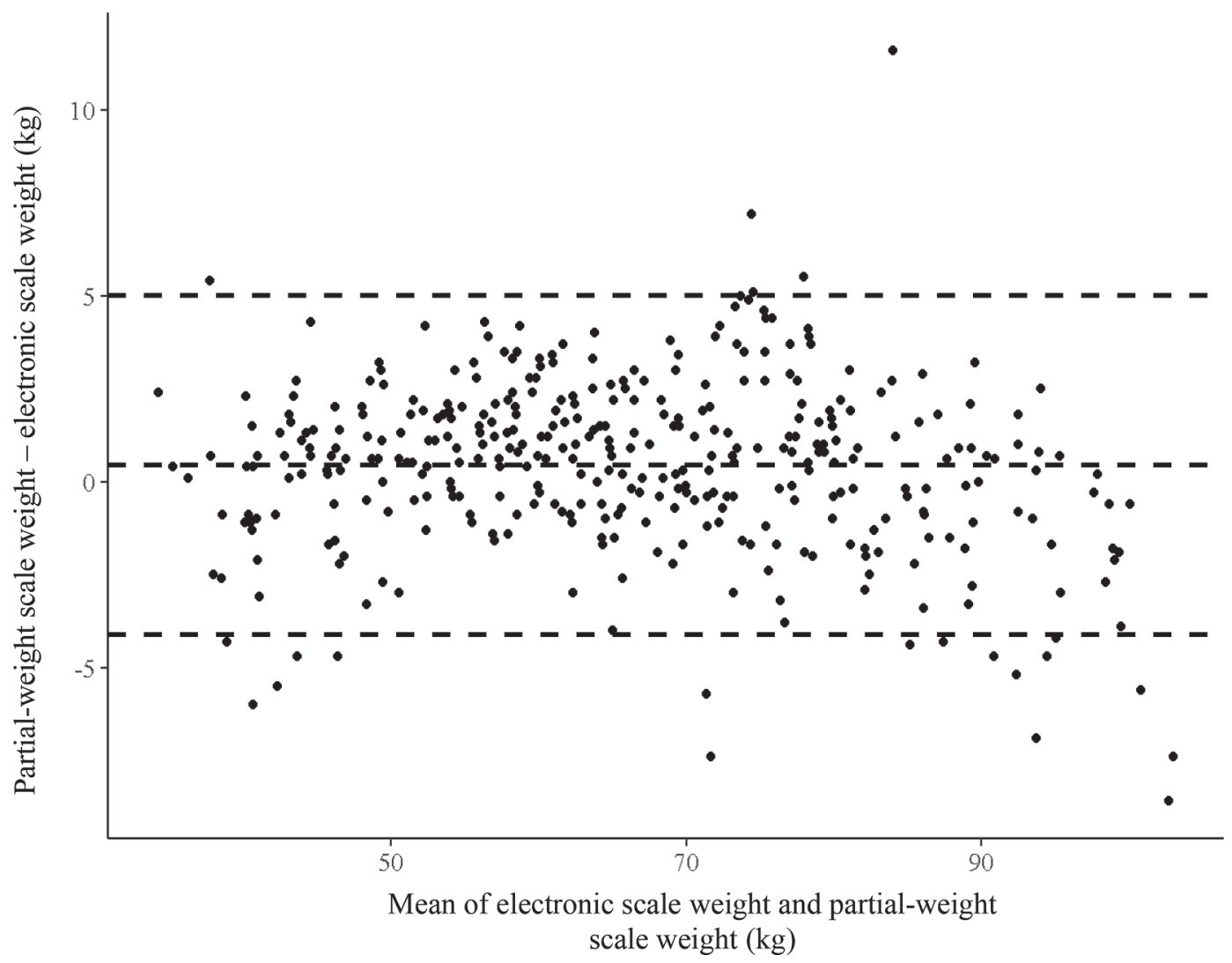

Figure 2. Bland-Altman plot of dairy heifer $(n=20)$ mean weights (376 observations) reported by the partial-weight scale attached to an automated milk feeder and the weights measured manually using an electronic scale (x-axis), and the difference between mean weights of the partial scale and electronic scale (y-axis). The center dashed line represents the mean difference, and upper and lower dashed lines represent the upper and lower levels of agreement. All heifers had 19 weights except for 1935 ( $\mathrm{n}=17), 1948(\mathrm{n}=18)$, and $1956(\mathrm{n}=18)$. 
(Clapham and Nicholson, 2009) and is critical for estimating the weight of calves. Low precision in a scale would potentially lower the effectiveness of a scale if a producer chose to evaluate nutritional performance during a specific time (e.g., weaning, postweaning), which was not the case in this study as we observed high precision in the partial-weight scale. Low precision could potentially create false alarms (Menzies et al., 2007). However, we observed high precision in the partial-weight scale. Research has shown that heart girth tapes had high precision for estimating calf weight compared with an electronic scale (Heinrichs et al., 2017). Because heart girth tapes still require manual handling of the calf, partial-weight scales may be attractive for producers implementing an automated milk feeding system. In addition, we observed high accuracy and low bias. High accuracy indicates how closely a technology is to the true measurement and has been suggested by engineers in conference proceedings to be essential for status monitoring (Ito et al., 1999). Indeed, high accuracy is critical for weight measurements that may be used by producers to determine appropriate medical dosage to treat sick animals (Wood et al., 2015). In addition, no influence of individual animals from this age group was observed. This contrasts with hipometers, which are reported to be reliable only for estimating weights of heifers greater than 3 mo and less than 15 mo of age (Dingwell et al., 2006). An advantage of the partial-weight scale used in this study is that it removes manual labor and human error associated with traditional weight estimates from hipometers and weight tapes. However, we validated this partial-weight scale only for calves up to $84 \mathrm{~d}$ of age. Thus, future research should determine whether the partial-weight scale is also valid for use in animals older than 3 mo.

Finally, we found a low bias, suggesting that the partial-weight scale is consistent in estimating weight as a calf ages even after weaning. Because most precision dairy technologies are used to monitor animal health, it is imperative that there is low bias, particularly for monitoring growth. Our results agree with the validation of heart girth tapes, in which our partialweight scale was accurate and precise and regressions were not influenced by individual calves as shown in our study (Heinrichs et al., 2017). However, heart girth tapes require manual labor, which is a likely barrier to adoption. In contrast, a partial-weight scale that automatically collects these data may be attractive to large

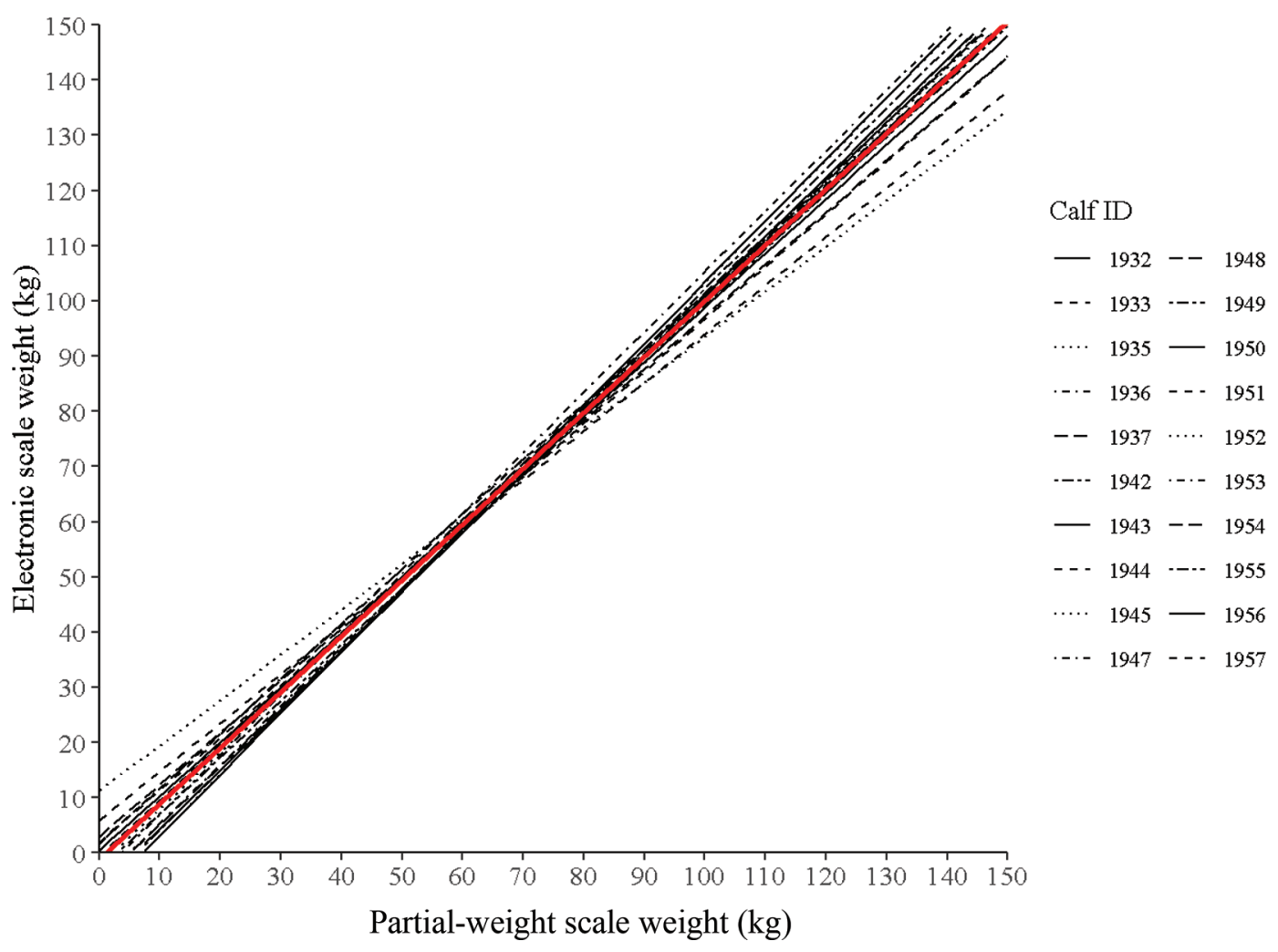

Figure 3. Regression of weights for each heifer $(\mathrm{n}=20)$ comparing the weight measured using the partial-weight scale (x-axis) against the weights recorded manually by the electronic scale (y-axis). All heifers had 19 weights except for 1935 (17 weights), 1948 (18 weights), and 1956 (18 weights). 
dairies, which are more likely to implement a precision technology (Gargiulo et al., 2018).

This partial-weight scale in this study required an algorithm to remove biologically irrelevant data; however, these observations were rare and removed by a single-step algorithm. In summary, we found the partialweight scale attached to an automated milk feeder to be very precise, accurate, and low in bias for estimating the weight of dairy calves, and it performed similarly to a manually reported electronic scale. To our knowledge, this is the first validation study of a partial-weight scale attached to an automated milk feeder for measuring the weight of dairy calves. Because this partial-weight scale was validated for both precision and accuracy, this partial-weight scale system may be useful for producers for estimating $\mathrm{BW}$ in calves in commercial settings, though the algorithm in this software could be improved by removing zero-weight measurements and weights lower than a calf's 3 -d weight average. However, we caution that the validation of this partialweight scale is associated with an algorithm specific to this automated milk feeder's software. Therefore, validation of a partial-weight scale is dependent on the software interpreting the calf's partial BW. In addition, we conducted this study on a research facility in a warm climate. Future research should determine the practicality of this partial-weight scale platform in colder climates in a commercial setting, where ice may cause calves to slip and become apprehensive about the scale. In summary, this scale was valid with low bias and may be useful for estimating daily BW in calves.

\section{ACKNOWLEDGMENTS}

The authors have not stated any conflicts of interest.

\section{REFERENCES}

Bach, A., and J. Ahedo. 2008. Record keeping and economics of dairy heifers. Food Anim. Pract. 24:117-138. https://doi.org/10.1016/j .cvfa.2007.10.001.
Clapham, C., and J. Nicholson. 2009. The Concise Oxford Dictionary of Mathematics. 4th ed. Oxford University Press, New York, NY.

Dingwell, R. T., M. M. Wallace, C. J. McLaren, C. F. Leslie, and K. E. Leslie. 2006. An evaluation of two indirect methods of estimating weight in Holstein calves and heifers. J. Dairy Sci. 89:3992-3998. https://doi.org/10.3168/jds.S0022-0302(06)72442-0.

Enevoldsen, C., and T. Kristensen. 1997. Estimation of body weight from body size measurements and body condition scores in dairy cows. J. Dairy Sci. 80:1988-1995. https://doi.org/10.3168/jds .S0022-0302(97)76142-3.

Gargiulo, J. I., C. R. Eastwood, S. C. Garcia, and N. A. Lyons. 2018. Dairy farmers with larger herd sizes adopt more precision dairy technologies. J. Dairy Sci. 101:5466-5473. https://doi.org/10 .3168/jds.2017-13324.

Heinrichs, A. J., B. S. Heinrichs, C. M. Jones, P. S. Erickson, K. F. Kalscheur, T. D. Nennich, B. J. Heins, and F. C. Cardoso. 2017. Verifying Holstein heifer heart girth to weight prediction equations. J. Dairy Sci. 100:8451-8454. https://doi.org/10.3168/jds .2016-12496.

Heinrichs, A. J., G. W. Rogers, and J. B. Cooper. 1992. Predicting body weight and wither height in Holstein heifers using body measurements. J. Dairy Sci. 75:3576-3581. https://doi.org/10.3168/ jds.S0022-0302(92)78134-X.

Hinkle, D. E. 1988. Applied Statistics for the Behavioral Sciences. 2nd ed. Houghton Mifflin, Boston, MA.

Ito, S., K. Hayasaki, K. Kawano, K. Sho, S. Mimotogi, F. Komatsu, and K. Okumura. 1999. High-accuracy development monitoring technology. Proc. SPIE 3743:142-150. https://doi.org/10.1117/12 .346907 .

Long, N. M., R. J. Collier, and J. F. Smith. 2012. Short communication: Comparison of 2 methods of assessing calf birth weights in dairy calves. J. Dairy Sci. 95:7206-7209. https://doi.org/10.3168/ jds.2012-6051.

Menzies, T., A. Dekhtyar, J. Distefano, and J. Greenwald. 2007. Problems with precision: A response to comments on "Data mining static code attributes to learn defect predictors." IEEE Trans. Softw. Eng. 33:637-640. https://doi.org/10.1109/TSE.2007.70721.

Moinester, M., and R. Gottfried. 2014. Sample size estimation for correlations with pre-specified confidence interval. Quantitative Methods Psychol. 10:124-130. https://doi.org/10.20982/tqmp.10 .2.p124.

Radostits, O. M., K. E. Leslie, and J. Fetrow. 1994. Herd Health: Food Animal Production Medicine. Saunders, Philadelphia, PA.

Wood, S., K. K. Reyher, and D. C. Barrett. 2015. Comparison of visual assessment and heart girth tape measurement for estimating the weight of cattle in clinical practice. Vet. J. 203:337-338. https: //doi.org/10.1016/j.tvjl.2014.12.034.

\section{ORCIDS}

Melissa C. Cantor @ https://orcid.org/0000-0002-4963-064X Joao H. C. Costa @ https://orcid.org/0000-0001-9311-4741 